\title{
Operational experience with nanocoulomb bunch charges in the Cornell photoinjector
}

\author{
Adam Bartnik, ${ }^{*}$ Colwyn Gulliford, ${ }^{\dagger}$ Ivan Bazarov, Luca Cultera, and Bruce Dunham \\ CLASSE, Cornell University, Ithaca, New York 14850, USA
}

(Received 3 April 2015; published 19 August 2015)

\begin{abstract}
Characterization of 9-9.5 MeV electron beams produced in the dc-gun based Cornell photoinjector is given for bunch charges ranging from $20 \mathrm{pC}$ to $2 \mathrm{nC}$. Comparison of the measured emittances and longitudinal current profiles to optimized 3D space charge simulations yields excellent agreement for bunch charges up to $1 \mathrm{nC}$ when the measured laser distribution is used to generate initial particle distributions in simulation. Analysis of the scaling of the measured emittance with bunch charge shows that the emittance scales roughly as the square root of the bunch charge up to $300 \mathrm{pC}$, above which the trend becomes linear. These measurements demonstrate that the Cornell photoinjector can produce cathode emittance dominated beams meeting the emittance and peak current specifications for next generation free electron lasers operating at high repetition rate. In addition, the 1 and $2 \mathrm{nC}$ results are relevant to the electron ion collider community.
\end{abstract}

DOI: 10.1103/PhysRevSTAB.18.083401

PACS numbers: 29.20.Ej, 41.75.Fr

\section{INTRODUCTION}

The development of a high-luminosity polarized electron-ion collider (EIC) with center of mass energies on the order of $100 \mathrm{GeV}$ represents the next major accelerator based nuclear physics experimental challenge [1]. Realization of such a facility requires significant advances in accelerator technology, including, but not limited to the development of energy recovery linacs (ERLs) with unprecedented currents and energies (up to multi-GeV) [2-5], as well as the development of both polarized and unpolarized electron sources beyond the state of the art in high-brightness, high-current machines. In particular, the production of multi-nanocoulomb electron bunches delivered at repetition rates up to tens of $\mathrm{MHz}$ represents a major technological hurdle for polarized electron source technology.

While not currently operating as a polarized source, the Cornell photoinjector provides a unique experimental setup for testing the feasibility of many of the design goals for EIC electron sources. In this paper, we demonstrate that the Cornell photoinjector is capable of delivering highbrightness beams with bunch charges up to $2 \mathrm{nC}$ (well beyond the initial injector design specification). In doing so, we give a more detailed description of the production of cathode emittance dominated beams which meet the design emittance and peak current specifications for the next

\footnotetext{
*acb20@cornell.edu

†cg248@cornell.edu
}

Published by the American Physical Society under the terms of the Creative Commons Attribution 3.0 License. Further distribution of this work must maintain attribution to the author $(s)$ and the published article's title, journal citation, and DOI. generation LCLS-II light source discussed in [6]. Comparison is given with optimized 3D space charge simulations for bunch charges up to $1 \mathrm{nC}$. These results help determine where the current state of the art in dc gun based photoinjectors stands with respect to EIC electron source goals, and provide further benchmarking of 3D space charge codes at bunch charges not previously explored.

The remainder of this paper is structured as follows: first we give an updated description of the injector layout. Next, a detailed account of the setup and calibration of the machine is given, including a description of the optimizations used to find the machine settings producing optimal emittance. In addition, the discovery and mitigation of stray quadrupole fields in the emittance compensation solenoids is discussed. After that follows the results of measurements of the emittance and longitudinal current profile for bunch charges from $20 \mathrm{pC}$ to $2 \mathrm{nC}$, and the corresponding comparison to $3 \mathrm{D}$ space charge simulations. Last, we discuss the effect of measured laser shape on the optimal emittances measured.

\section{EXPERIMENTAL SETUP}

Figure 1 shows the current layout of the Cornell photoinjector. The element positions in the gun and linac sections of the injector remain identical to those used in previous measurements $[7,8]$. However, the diagnostic beam lines after the superconducting radio frequency (SRF) cryomodule have changed substantially during the subsequent years. Currently, the merger section used in previous emittance measurements has been removed, and the entrance to the chicane section has been moved upstream towards the SRF cryomodule. The double slit emittance measurement system (EMS) is now located in the straight section, in 


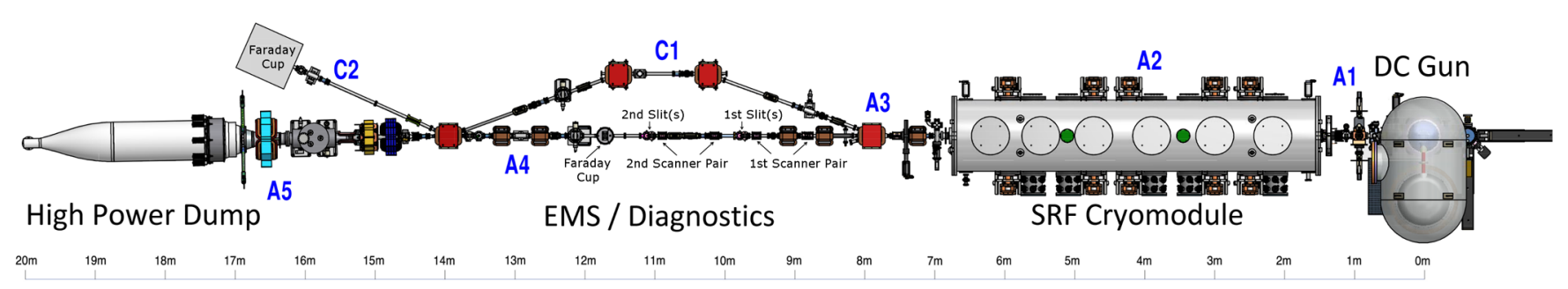

FIG. 1. Top view of the Cornell injector. The deflecting cavity (not shown) is located between the second EMS slit pair and the A4 Faraday cup.

between the entrance and exit of the chicane, with three quadrupole magnets before the first measurement slit. New radiation shielding was added around the EMS so that emittance measurements could be performed at energies above roughly $7 \mathrm{MeV}$ [7], allowing for neutron production. The dc gun has now been processed up to $395 \mathrm{kV}$, but all other features of its design remain the same $[9,10]$. Similarly, the buncher has been processed up to $90 \mathrm{kV}$, to allow for adequate bunching at high charge. No additional processing was needed for the five SRF cavities, running stably up to 2-2.5 MV energy gain. For this paper, a NaKSb cathode with a mean transverse energy (MTE) of $140 \pm 10 \mathrm{meV}$ and a large $(>1 \mathrm{~cm})$ active area with a nearly uniform quantum efficiency $(\mathrm{QE})$ around $5 \%$ was used [11]. A more detailed description of the beam line and EMS can be found in $[7,12]$ and $[9,13]$ respectively.

\section{EXPERIMENT}

\section{A. Setup and calibration}

\section{Mutiobjective genetic algorithm optimizations}

In order to find machine states producing optimal emittance compensation for each bunch charge, we use multiobjective genetic algorithm (MOGA) optimization software and the 3D space charge code general particle tracer (GPT) [14] (a detailed description of our 3D injector model can be found in [7]). For each of the desired charges up to $1 \mathrm{nC}$ (issues with $2 \mathrm{nC}$ are discussed later), we simultaneously optimized both the emittance and rms bunch length at the location of the EMS in the simulated injector, subject to physical constraints on all relevant injector and beam parameters. In contrast to previous simulations [7], in these optimizations the optimizer varied the initial laser distribution by selecting the laser pinhole diameter, truncation fraction, and the longitudinal shaping crystal angles, in precisely the same manner that the real laser is adjustable.

For all bunch charges, the optimizer selected a final energy of roughly 9-9.5 MeV. At this energy, the emittance continues to oscillate due to space charge after exiting the injector cryomodule. Consequently, the optimizer chose settings producing an emittance oscillation minimum at the location of the EMS, roughly three meters downstream of the rf cavities. For bunch charges up to $300 \mathrm{pC}$, the resulting optimized injector settings satisfied all of the LCLS-II injector 95\% emittance and peak current specifications. To accomplish this, the optimizer chose laser pinhole sizes of $0.73,1.9$, and $3.5 \mathrm{~mm}$, as well as a truncation of roughly $50 \%$ for each respective charge. Moving well above those charges in simulation began to necessitate unrealistically large transverse laser distributions, and as a result, we switched to using a longer set of shaping crystals in simulation for charges above $300 \mathrm{pC}$. With this change, similar optimizations were carried out for $1 \mathrm{nC}$. As with the LCLS-II bunch charges, the optimizer selected roughly a 50\% truncation fraction for the transverse laser distribution and roughly a $5 \mathrm{~mm}$ pinhole size. Simulations were not carried out for $2 \mathrm{nC}$, though one would expect the ideal aperture size to scale to approximately $7 \mathrm{~mm}$, as it scales roughly as $\sqrt{q}$, keeping the peak charge density constant.

\section{Laser modifications and characterization}

For all measurements in this paper, we exclusively use a $50 \mathrm{MHz}$ laser, as well as a pulse picking Pockels cell to reduce the average power deposited in the interceptive EMS. At this repetition rate, a $2 \mathrm{nC}$ bunch charge would require 5 Watts of laser power for a $5 \% \mathrm{QE}$ cathode. Allowing for losses in transport and shaping, this requires $>10 \mathrm{~W}$ of initial laser power. As a result, the laser was recently upgraded [15] to produce up to $70 \mathrm{~W}$ average power, however, in order to remain below the manufacturer's specified average power damage threshold of the Pockels cell, the average power was limited to $20 \mathrm{~W}$. With no transverse shaping (i.e., clipping) of the laser beam, $2.5 \mathrm{nC}$ bunches were produced at the maximum allowable laser power.

The shape of the laser is tunable both in the transverse and longitudinal directions. For longitudinal shaping, $N$ rotatable birefringent crystals $\left(\mathrm{YVO}_{4}\right)$ temporally shape the primary pulse by splitting it into $2^{N}$ copies with tunable relative intensities set by the crystals' rotation angles. As in simulation, we used two sets of crystals for this work, the longer set used for charges above $300 \mathrm{pC}$. This results in a roughly 9 and $25 \mathrm{ps}$ rms laser pulse length for bunch charges of 20-300 pC, and 1-2 $\mathrm{nC}$, respectively. The crystal lengths for both sets are given in Table I. 
TABLE I. Longitudinal shaping crystal lengths.

\begin{tabular}{lc}
\hline \hline Bunch charge & $\mathrm{YVO}_{4}$ crystal lengths $(\mathrm{mm})$ \\
\hline $0-300 \mathrm{pC}$ & $\begin{array}{r}15.096,7.5480,3.7740,1.8870 \\
\text { (four crystal set) }\end{array}$ \\
$1 \mathrm{nC}, 2 \mathrm{nC}$ & $40.8,20.4,10.2,5.1,2.55$ (five crystal set) \\
\hline \hline
\end{tabular}

For transverse shaping, we use a manually adjustable beam expander and pinhole aperture [16] to clip the approximately Gaussian laser distribution at roughly the half maximum intensity. This amount of truncation of the laser was found to work consistently well in simulation for all charges. Importantly, truncating a Gaussian beam at the $50 \%$ intensity point also clips off half of the laser power. Since we only had enough laser power to produce $2.5 \mathrm{nC}$ with no transverse clipping, we could not clip off $50 \%$ and still reach $2 \mathrm{nC}$. For this bunch charge, a $8.8 \mathrm{~mm}$ aperture (compared to the $7 \mathrm{~mm}$ optimal value) and less aggressive clipping was required. Using the simulation as a guide, the diameter of the laser was chosen from the available set of pinhole apertures. Notably, with the exception of the smallest aperture, all of the available pinholes were within a few percent of the simulation values for charges up to $1 \mathrm{nC}$. For the smallest pinhole a $1 \mathrm{~mm}$ aperture was used instead of the $0.73 \mathrm{~mm}$ size selected by the optimizer, leading to a slightly larger thermal emittance than is optimal.

For each emittance measurement, the transverse shape of the laser beam was recorded by picking off the laser beam and sending it to a CCD at precisely the same path length as the cathode. The longitudinal shape was measured once for each crystal set by sending a near-zero charge beam $(0.02 \mathrm{pC})$ through the injector with all cavities turned off. Because there is very little effect from space charge at this charge, the temporal profile remains nearly constant throughout the machine. After reaching the diagnostics section, the beam was passed through a single horizontal slit of the EMS, producing a thin beamlet on a downstream viewscreen. The width of that slit $(20 \mu \mathrm{m})$, as well as the timing jitter of the laser with respect to the deflecting cavity (300 fs rms), determines the measurement resolution. Turning on the vertical deflecting cavity after the slit resolves the time axis of the beamlet on the downstream viewscreen, providing a measurement of the temporal beam profile.

The transverse profiles recorded during each emittance measurement and the longitudinal profiles for both crystal sets are shown in Fig. 2, along with a comparison to a longitudinal simulation done with the $0.02 \mathrm{pC}$ machine settings. Interestingly, even though the temporal profile of the laser beam has many sharp peaks due to the stacking of individual laser pulses, by the time the bunch reaches the deflector, these peaks are smeared out from space charge both in simulation and measurement, even at this small bunch charge.
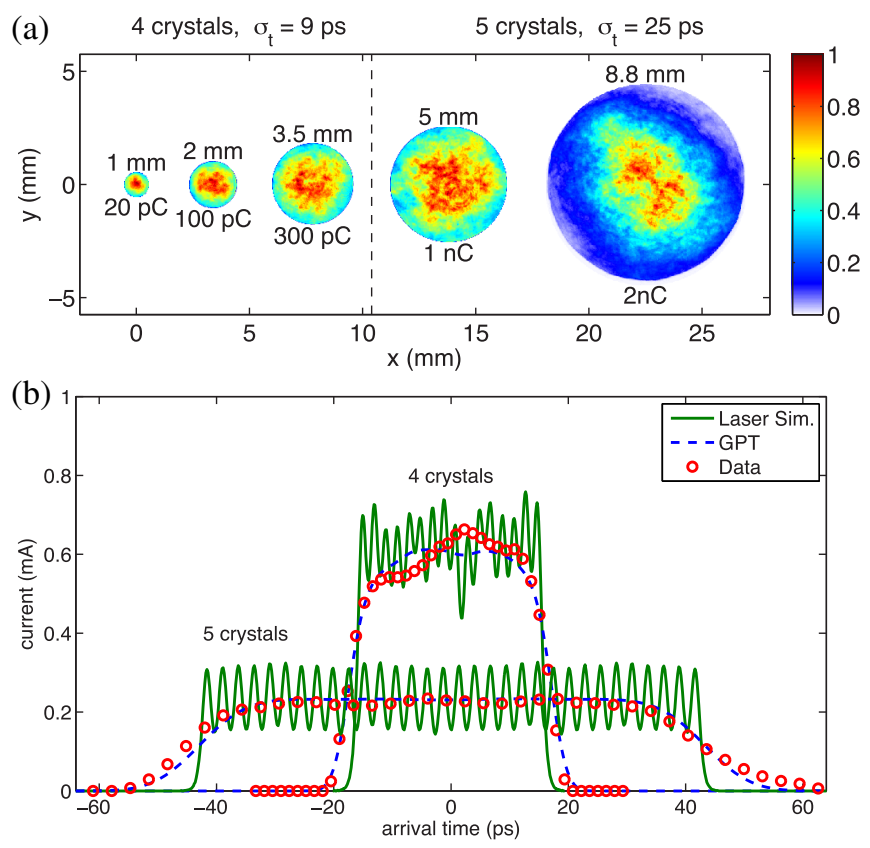

FIG. 2. Characterization of the laser distribution: (a) measured transverse laser distributions; (b) simulated temporal laser shape (green) and corresponding simulated (blue) and measured (red) temporal electron current profile at the EMS with no rf.

The pulse picking Pockels cell is not fast enough to pick a single laser pulse, so a short pulse train is used for emittance measurements. In practice, this sets a minimum duration of $300 \mathrm{~ns}$ to the bunch train, long compared to the rise and fall time of the Pockels cell. Below this minimum duration, the rise and fall time cause a significant portion of the bunches in the bunch train to have the wrong charge, confusing the interpretation of the measured phase spaces. Longer durations were used for smaller bunch charges, in order to increase the signal level from the EMS. At the largest charges, this minimum duration causes there to be significant beam loading in the cavities, especially in the buncher. At $1 \mathrm{nC}$, the buncher phase was seen to vary by roughly 5 degrees (peak to peak) during the bunch train, and almost 10 degrees at $2 \mathrm{nC}$. Somewhat surprisingly, reducing the duration of the bunch train by a factor of 2 did not affect the measured phase spaces, and thus the optimal machine settings are apparently robust to that level of buncher phase changes. To roughly estimate this effect in simulation, we offset the buncher cavity phase by $2.5 \mathrm{deg}$ for the $1 \mathrm{nC}$ bunch charge settings in GPT and saw a roughly $10 \%$ increase/decrease in the horizontal/vertical emittance respectively. This error is roughly within the systematic error of the EMS.

\section{EMS calibration and thermal emittance}

The largest source of error in the EMS measurements is the calibration of the scanner magnets. Using a downstream 


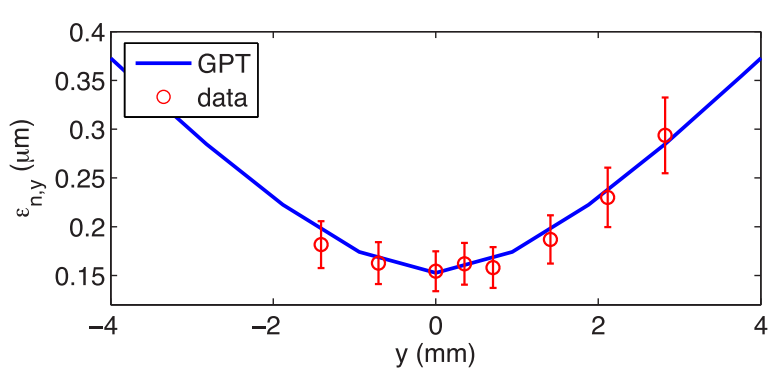

FIG. 3. Measured and simulated vertical emittance as a function of misalignment going into the first SRF cavity, set on crest at $1500 \mathrm{kV}$ cavity voltage. All other SRF cavities were off.

viewscreen, the EMS scanner magnets were calibrated at the target energy of $9 \mathrm{MeV}$. To verify this calibration, the emittance of the beam at near zero charge was measured using the EMS and compared to the value at the cathode, computed using Eq. (1) and the laser distribution measured by diverting the laser beam to a CCD. Both values agreed within a few percent:

$$
\epsilon_{\text {cathode }}=\sigma_{\text {laser }} \sqrt{\frac{\mathrm{MTE}}{m_{e} c^{2}}} .
$$

Once calibrated, the EMS measurement of the thermal emittance is a valuable tool to verify the alignment of the various optics in the beam line. Without the effect of space charge, the emittance of the beam is conserved along the beam line, unless one has aberrations from misaligned optics. As both another check of the EMS system itself, and also to verify the cavity field maps used in GPT, we intentionally misaligned the beam through the first SRF cavity using the last steering magnet before it, keeping all other cavities turned off. In simulation, we modeled the same situation, including a model of the steering magnet. As seen in Fig. 3, the thermal emittance grows just as expected from simulation.

(a)

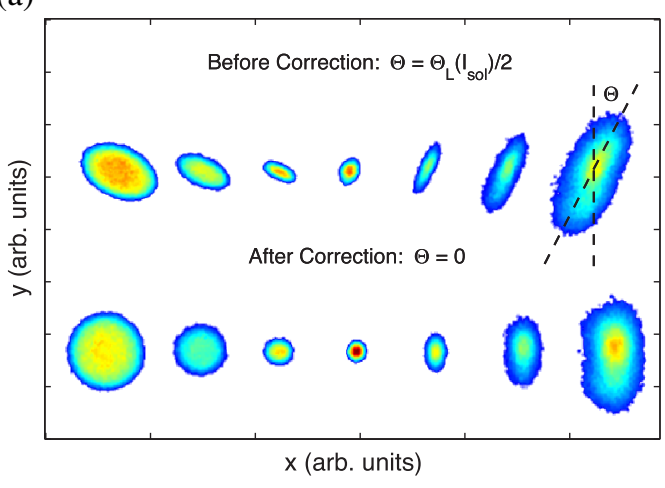

\section{Stray quadrupole fields in the solenoid}

Initial measurements at $20 \mathrm{pC}$ produced asymmetric horizontal and vertical emittance values, similar to those measured in the merger section [7]. Previously, this asymmetry was suspected to be due to the horizontal bends that the beam takes in the merger, however this could no longer explain the asymmetry seen in the straight section. We initially thought the asymmetry was due to misalignment in the gun, solenoids, buncher, or first two SRF cavities. Subsequent attempts at realigning the beam through these elements did not reduce the asymmetry. Further investigation lead to the discovery of an asymmetric beam spot on the first viewscreen (for bunch charges with at least $10 \mathrm{pC}$ ) when the solenoid was tuned to put the beam near a focus on the first viewscreen. Below that charge, due to the optics layout, the beam could not be strongly focused, and the asymmetry was not noticeable to the eye.

Varying the solenoid current changed not only the size of the elliptical beam spot on the viewscreen, but also the orientation of the ellipse. We also noticed that the semimajor axes of the elliptical beam profile aligned perfectly with the kick axes of the horizontal and vertical corrector magnets at the solenoid center, suggesting the ellipse orientation was exactly half of the Larmor angle, as shown in Fig. 4(a). This suggested the presence of a stray quadrupole moment at the solenoid center [17].

Modeling this effect in GPT allowed for the estimation of the stray quad strength by fitting to the measured transverse second moments of the beam on the first viewscreen as a function of the solenoid current. Figure 4(b) displays the results of the fits. Assuming a 3 inch effective quad length resulted in a roughly $0.5 \mathrm{G} / \mathrm{cm}$ quadrupole gradient at a solenoid current around $3 \mathrm{~A}$. By repeating this fitting procedure at different gun voltages (allowing the beam to focus at different solenoid currents), and also by checking the other polarity of the solenoid magnet, we were able to

(b)
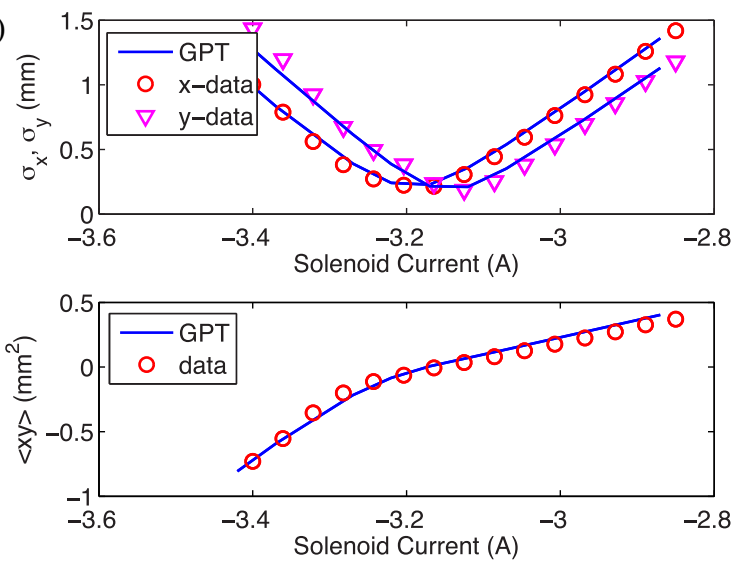

FIG. 4. Characterization and correction of the quadrupole moment in the first solenoid. (a) Elliptical beam spot on the first viewscreen after the first solenoid. The tilt of the ellipse was exactly half the Larmor angle of the solenoid. (b) Fitting the measured second moments of the beam spot on the first viewscreen to GPT to estimate the quad strength. 

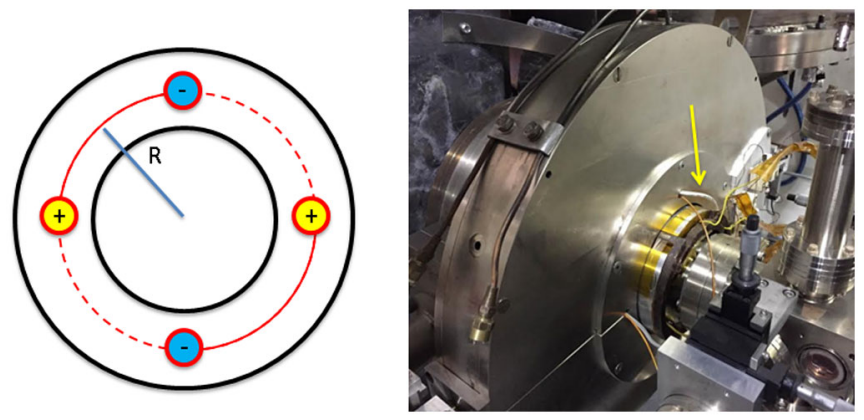

FIG. 5. Wiring of the correction quadrupole magnet, and photo of the wiring as installed. The single turn of wire is indicated with an arrow.

verify that the strength of the quad field scales linearly with the applied solenoid current.

A temporary correction to this problem was found by using the available holes in the solenoid magnet frame, originally intended as beam position monitor (BPM) wire feedthroughs, to wire a single-turn correction quad. The field from this type of magnet may be roughly estimated using

$$
\begin{aligned}
& B_{x}=\frac{2(N I) \mu_{0}}{\pi R^{2}} y \\
& B_{y}=\frac{2(N I) \mu_{0}}{\pi R^{2}} x .
\end{aligned}
$$

From these expressions, it was estimated that roughly 40 Amp-turns of coil are required to cancel the field given the coil radius of 6 inches, and a length of 3 inches. Correcting coils were wired through both solenoids in the injector, as seen in Fig. 5.

We set the correcting quad coil power supply to scale automatically with the solenoid power supply, with an adjustable scale factor determined by making the beam as round as possible on the viewscreen. Figure 4(a) shows the resulting beam spots after correction. The corrector coils successfully removed the solenoid dependent tilt of the beam spot, effectively a skew quad, however we still note some remaining $\mathrm{x}-\mathrm{y}$ asymmetry in the beam spot, perhaps due to a (normal) quadrupole field from the downstream ion pump. We did not attempt to correct this additional remaining stray field, hoping that the effect of this nonskew quad might be canceled by adjusting the downstream quads in the measurement section.

The same fitting procedure was not done for the second solenoid because the nearest viewscreen is located after the SRF cavities, over $6 \mathrm{~m}$ downstream, a distance too far to allow for a reliable measurement at $395 \mathrm{kV}$ beam energy. For this magnet we used roughly the same scale factor with solenoid current to determine its correcting coil current, but allow this parameter to be adjusted while searching for minimum emittances.

\section{Faraday cup selection}

The injector has two Faraday cups that can be used to collect the charge during emittance measurements: one in the middle of the A4 section after the second EMS slit and one after the $20^{\circ}$ bend in the $\mathrm{C} 2$ section. Ideally, if all of the charge is correctly guided to either Faraday cup, the measured phase space should be independent of which Faraday cup is used, as the same EMS slits in the A4 sections are used to clip the beam in both cases. In practice however, we found a systematic discrepancy between subsequent measurements using both Faraday cups.

Figure 6 shows an example phase space measured using both the A4 and C2 Faraday cups. The majority of the phase space is identical in both cases, but when using the A4 Faraday cup, there is always a vertical smearing, producing a shadowlike background mostly below the core of the phase space. We were unable to determine the cause of this, but believe this to be an artifact of the measurement. We tried changing which axis of the phase space is scanned quickly, to see if the smearing is somehow due to the rapid changing of the scanner magnets, but the background was always in the same place. We verified that there was no beam lost when sending the beam into the $\mathrm{C} 2$ Faraday cup by moving all corrector dipoles over large ranges, and seeing no change in the measured phase space. Performing a phase space measurement with the electron beam off shows only noise, implying that dark current from the rf cavities is not the source of the background, as this would be present without the beam. We speculate that the spurious background is due to secondary electrons produced in the Faraday cup from radiation that penetrates the second EMS slit. This slit does not have the same armor beamstop material around the slit opening like the first slit [9], and would be more likely to have radiation leaking through it. Bending the remaining electrons into the $\mathrm{C} 2$ section would separate the electrons from the radiation and thus remove the background. Unless otherwise stated, all phase spaces reported here were measured using the Faraday cup in the $\mathrm{C} 2$ section.

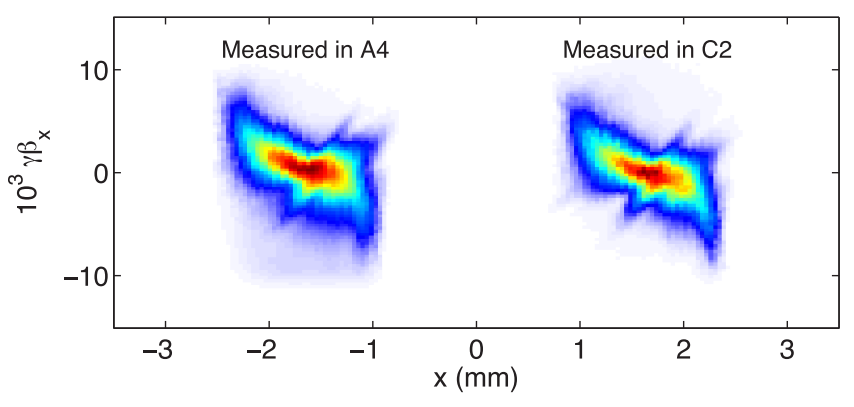

FIG. 6. Comparison of the measured phase space using the A4 and C2 Faraday cups. The $100 \%$ emittance in both images is 0.802 and $0.785 \mu \mathrm{m}$, respectively. 
TABLE II. Injector settings.

\begin{tabular}{|c|c|c|c|c|c|}
\hline Parameter & $20 \mathrm{pC}$ & $100 \mathrm{pC}$ & $300 \mathrm{pC}$ & $1 \mathrm{nC}$ & $2 \mathrm{nC}$ \\
\hline Laser pinhole, rms bunch length (mm, ps) & 1,8 & 2,8 & $3.5,8$ & 5,25 & 8,25 \\
\hline Solenoid $1(\mathrm{~T})$ & -4.13 & -4.04 & -3.98 & -4.0 & -3.84 \\
\hline Buncher voltage, phase $(\mathrm{kV}$, deg) & $63,-90$ & $64,-90$ & $85,-90$ & $85,-90$ & $85,-90$ \\
\hline Solenoid 2 (T) & 2.18 & 2.33 & 2.58 & 2.86 & 2.98 \\
\hline SRF cavity 1 voltage, phase $(\mathrm{kV}$, deg) & $2100,-10$ & $2100,-10$ & $2100,-10$ & 1900,0 & 1900,0 \\
\hline SRF cavity 2 voltage, phase $(\mathrm{kV}$, deg) & $1000,-20$ & $1000,-20$ & $1000,-20$ & $1000,-15$ & $1000,-15$ \\
\hline SRF cavity 3 voltage, phase $(\mathrm{kV}$, deg) & $2300,-10$ & $2300,-10$ & $2300,-10$ & $2300,-10$ & $2300,-10$ \\
\hline SRF cavity 4 voltage, phase $(\mathrm{kV}$, deg) & $1700,-10$ & $1700,-10$ & $1700,-10$ & $1700,-10$ & $1700,-10$ \\
\hline SRF cavity 5 voltage, phase $(\mathrm{kV}$, deg) & $2000,-30$ & $2000,-30$ & $2000,-30$ & $2000,-30$ & $2000,-30$ \\
\hline A3 quad 1 (A) & -1.5 & -1.5 & -1.5 & -1.5 & -1.5 \\
\hline A4 quad 2 (A) & 1.5 & 1.5 & 1.5 & 1.5 & 1.5 \\
\hline
\end{tabular}

\section{B. Bunch charges up to $\mathbf{2} \mathbf{n C}$}

\section{Emittance measurements}

We began emittance measurements by loading the optimized machine settings from simulation for $20 \mathrm{pC}$ into the injector, and then tuning the machine for best emittance. During tuning, the horizontal and vertical emittance were rarely symmetric, and we would often improve one axis, only to find the other had worsened. Eventually, we found quad settings for the second solenoid corrector quad, and the first two normal quads in the A3 section that produced a roughly symmetric beam profile and symmetric emittances at the measurement slit. Note that the first corrector quad was held fixed during this process at the value that removed the stray field in the first solenoid. We expect that these optimized quad settings are not unique, and moreover likely depend on the SRF cavity settings, since there is a time dependent quadrupole effect from the couplers in the cavities. After settling on these SRF and quad settings, they did not need to be changed as the bunch charge was increased.

Instead, we found that simply changing the size of the initial laser profile, small tweaks to the first solenoid, and increasing the second solenoid and buncher voltage with
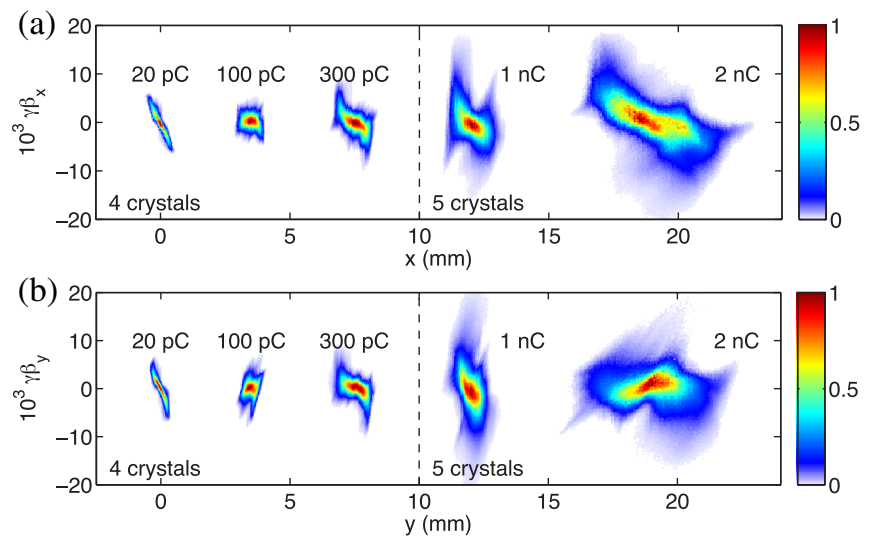

FIG. 7. Measured horizontal (a) and vertical (b) phase spaces as a function of bunch charge. charge were the only required changes up through $300 \mathrm{pC}$. Above that charge, we were no longer able to easily increase the buncher voltage, as we had reached the maximum level that it had been processed to. As a result, the bunch length entering the first cavity was longer than it had been previously, and perhaps due to this, we found that we had to slightly turn down its voltage and put it on crest for best emittance. The other SRF settings remained constant. All of these settings are summarized in Table II.

The final phase spaces and corresponding emittances for this paper are found in Fig. 7 and Table III, respectively. All phase spaces were produced using an $N \times N$ scan of the scanner magnet currents. In order to ensure the emittance was not overestimated due to pixelation of the phase spaces required $N \geq 100$. The EMS magnets scan at the same rate as the Pockels cell, typically $0.5-1 \mathrm{kHz}$. This corresponds to scan times of about 20 to $90 \mathrm{sec}$ depending on the number of scan points and Pockels cell rate used. This implies that each pixel in phase space is computed from the integrated charge over the Pockels cell macropulse. The Pockels cell duration for each phase space scan is adjusted to maximize the signal on the Faraday cup without saturating the electronics. Thus larger bunch charges have shorter Pockels cell duration: 10 to $0.3 \mu \mathrm{s}$ for $20 \mathrm{pC}$ to $2 \mathrm{nC}$.

Figure 8 shows emittance vs bunch charge trend. Though the emittance initially scales with the expected $\sqrt{q}$ dependence, above $300 \mathrm{pC}$ the trend becomes more linear with charge. Similarly, below $1 \mathrm{nC}$ the final $95 \%$ emittance is

TABLE III. Measured horizontal (vertical) normalized emittances $(\mu \mathrm{m})$.

\begin{tabular}{lllll}
\hline \hline $\mathrm{Q}(\mathrm{pC})$ & \multicolumn{1}{c}{$100 \% \epsilon_{n}$} & \multicolumn{1}{c}{$95 \% \epsilon_{n}$} & \multicolumn{1}{c}{ Core $\epsilon_{n}$} & Cathode $\epsilon_{n}$ \\
\hline 20 & $0.22(0.24)$ & $0.18(0.19)$ & $0.09(0.08)$ & $0.12(0.11)$ \\
100 & $0.37(0.39)$ & $0.30(0.32)$ & $0.16(0.16)$ & $0.24(0.23)$ \\
300 & $0.78(0.78)$ & $0.62(0.60)$ & $0.30(0.28)$ & $0.42(0.41)$ \\
1000 & $2.3(2.3)$ & $1.6(1.6)$ & $0.56(0.58)$ & $0.59(0.60)$ \\
2000 & $6.4(5.4)$ & $4.4(4.0)$ & $1.6(1.3)$ & $0.88(0.90)$ \\
\hline \hline
\end{tabular}




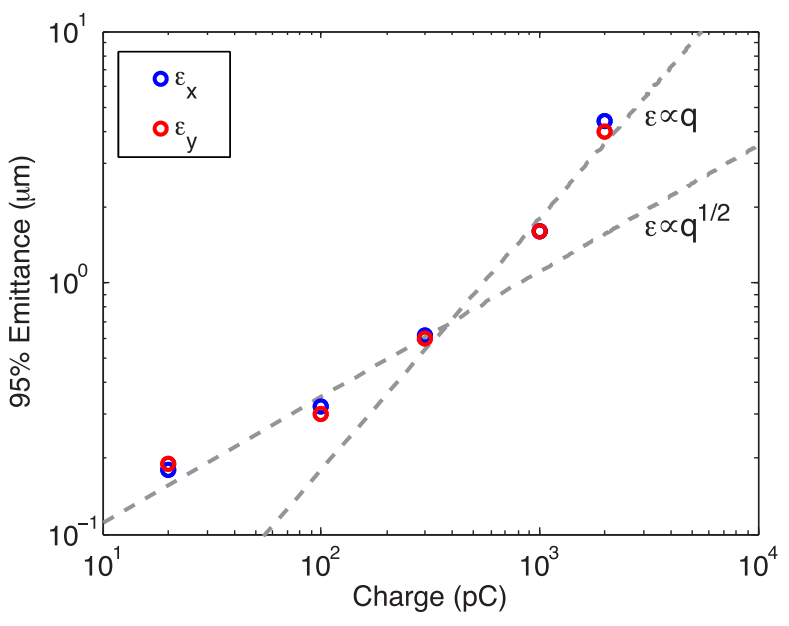

FIG. 8. Trend of the emittance as function of bunch charge.

only 1.5 times larger than the emittance on the cathode, but this grows to almost a factor of 5 for $2 \mathrm{nC}$. Clearly, the emittance growth is not as well compensated for the largest charges.

\section{Longitudinal current profiles}

Measuring the bunch longitudinal profile is more challenging at nonzero charge. For (near) zero charge, we would use the first EMS slit to select a narrow slice of the incoming beam, and then use the downstream deflector to spread that narrow slice out according to its temporal structure. An inherent assumption in this method is that all slices of the beam have the same temporal profile. At zero charge and with no rf, this assumption is valid, but in general this is not true. At higher charges, we instead perform a slice emittance measurement, effectively measuring the temporal profile individually for each pixel of the bunch's phase space, and then performing a weighted
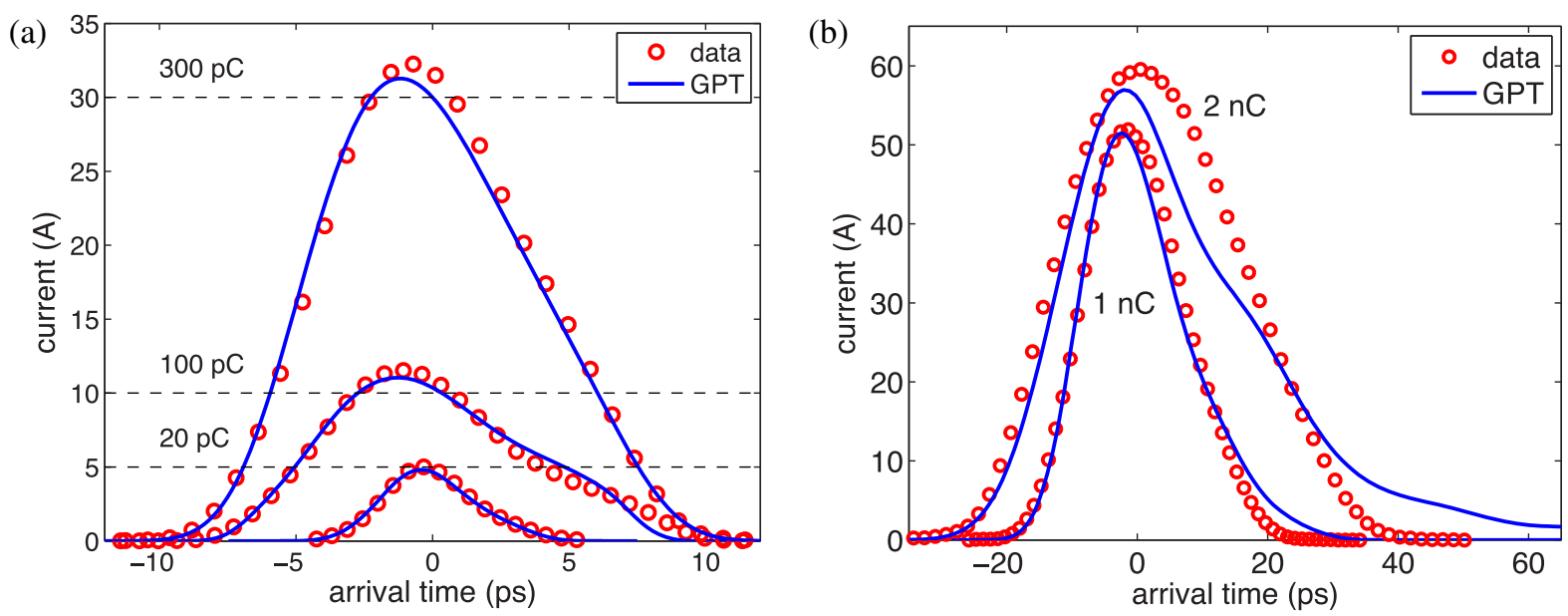

FIG. 9. The simulated and measured current profiles for each bunch charge.
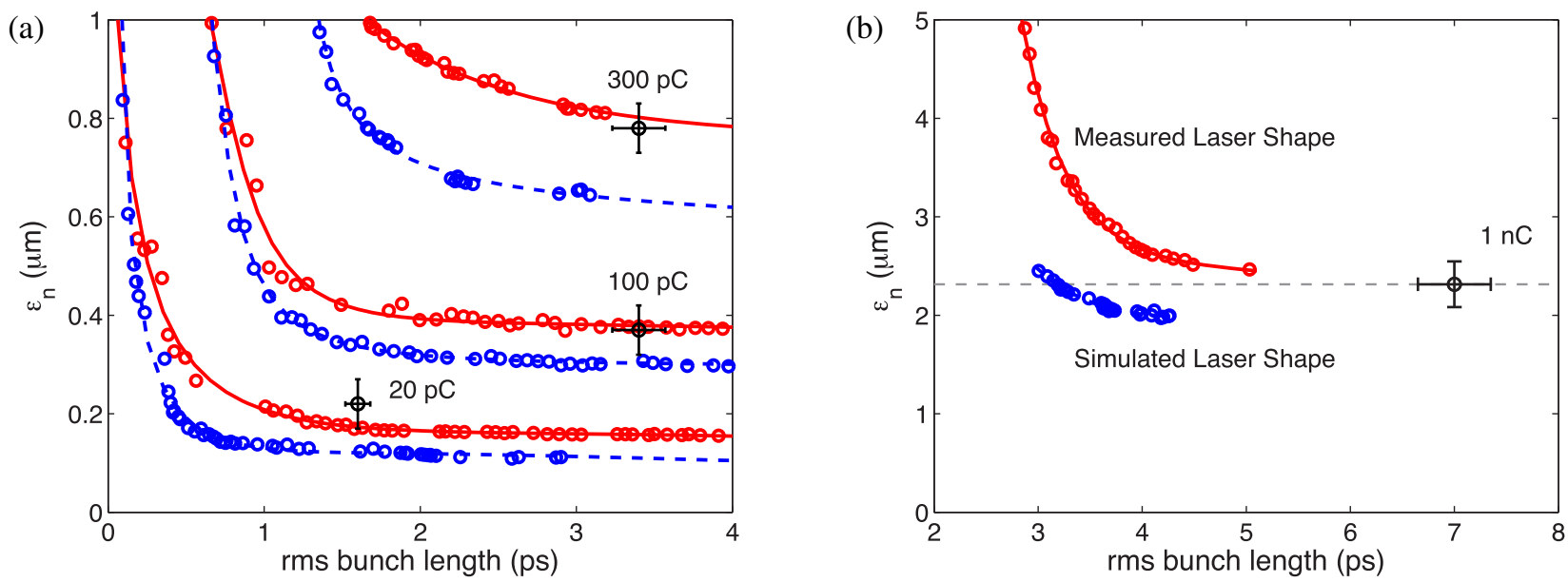

FIG. 10. Comparison of the optimal average 100\% transverse emittance for 20-300 pC bunch charges (a) and $1 \mathrm{nC}$ bunch charge (b). Shown in blue are the optimizations using an ideal, varied laser shape. In red are the optimization results using the measured transverse laser profile and fix shaping crystal angles. Measured values are shown in black. 
average to construct the profile for the entire bunch. All profiles shown in Fig. 9 were constructed in this manner. From these profiles, the rms bunch lengths at the EMS were computed. This gave 1.6, 3.4, and 3.4 ps for the LCLS-II charges, as well as 7 and 12 ps for the 1 and $2 \mathrm{nC}$ data. This corresponds to a compression factor of roughly $6,3,3,4$, and 2 for these charges respectively.

Also shown in Fig. 9 is a comparison to simulation at the same machine setting as used in each measurement. The simulation profiles have been smoothed using a Gaussian convolution, in order to smooth over the inherent noisiness from histogramming the particles' arrival times. Below $1 \mathrm{nC}$ the agreement is very good, but above that charge a long tail appears in simulation that is not measured in the actual machine.

\section{Effects of the laser shape}

In order to determine the effect that the initial transverse laser shape has on the final emittance, a second round of MOGA optimizations were performed at each bunch charge. In these simulations, the initial measured laser distributions shown in Fig. 2(a) were used to generate the transverse positions of the initial particle distributions. Additionally, the simulated longitudinal shaping crystals were set to produce the rough flattop distributions shown in Fig. 2(b). As previously mentioned, analysis of the first round of optimization results demonstrates that the final optimal emittances show a weak dependence on the longitudinal shaping crystal angles.

Figures 10(a) and 10(b) show the optimized average $100 \%$ transverse emittance $\epsilon_{n}=\frac{1}{2}\left(\epsilon_{n, x}+\epsilon_{n, y}\right)$ and $\mathrm{rms}$ bunch length computed from the initial optimizations with varied laser parameters (blue), computed from the second round of optimizations using the measured transverse laser profiles and fixed crystal angles (red), and computed from the phase space data taken with the EMS (black) for charges up to $300 \mathrm{pC}$ and $1 \mathrm{nC}$, respectively. Good agreement is seen in all cases when comparing the measured emittance values to those resulting from the simulations using the measured laser shape.

\section{CONCLUSION}

In this paper, the production of low emittance beams with normalized transverse emittance at $9-9.5 \mathrm{MeV}$ beam energy has been characterized for bunch charges up to 2 nC. MOGA simulations producing optimal emittances were used to determine the injector settings for all measurements. Realizing the final low emittances measured required the identification and correction of stray quad fields in the emittance compensation solenoids. Comparison of the measured emittances to optimized 3D space charge simulations shows excellent agreement when the measured laser distribution for each bunch charge is used to generate the initial particle distribution in simulation. Both the final measured emittances as well as the longitudinal current profiles show good agreement with simulation for bunch charges up to $1 \mathrm{nC}$. Additionally, for these charges the measured emittances were symmetric. For $2 \mathrm{nC}$ bunch charges, the emittance was likely nonoptimal as there was not sufficient laser power to clip the laser at the truncation fraction and pinhole size suggested by optimizations.

\section{ACKNOWLEDGMENTS}

This work was supported, in part, by the LCLS-II Project and the U.S. Department of Energy, Contract No. DEAC02-76SF00515, as well as DOE Nuclear Physics Award No. DE-SC0012493.

[1] http://arxiv.org/abs/1212.1701.

[2] eRHIC, A future electron-ion collider at BNL, in Proceedings of the 9th European Particle Accelerator Conference, Lucerne, 2004 (EPS-AG, Lucerne, 2004).

[3] http://arxiv.org/abs/1409.1633.

[4] http://arxiv.org/abs/1305.2090.

[5] http://lhec.web.cern.ch/.

[6] C. Gulliford, A. Bartnik, I. Bazarov, B. Dunham, and L. Cultrera, Appl. Phys. Lett. 106, 094101 (2015).

[7] C. Gulliford, A. Bartnik, I. Bazarov, L. Cultrera, J. Dobbins, B. Dunham, F. Gonzalez, S. Karkare, H. Lee, H. Li, Y. Li, X. Liu, J. Maxson, C. Nguyen, K. Smolenski, and Z. Zhao, Phys. Rev. ST Accel. Beams 16, 073401 (2013).

[8] B. Dunham et al., Appl. Phys. Lett. 102, 034105 (2013).

[9] I. V. Bazarov, B. M. Dunham, C. Gulliford, Y. Li, X. Liu, C. K. Sinclair, K. Soong, and F. Hannon, Phys. Rev. ST Accel. Beams 11, 100703 (2008).

[10] B. M. Dunham, C. K. Sinclair, I. V. Bazarov, Y. Li, X. Liu, and K. W. Smolenski, in Proceedings of the 22nd Particle Accelerator Conference, PAC-2007, Albuquerque, NM (IEEE, New York, 2007), pp. 1224-1226.

[11] L. Cultrera, S. Karkare, B. Lillard, A. Bartnik, I. Bazarov, B. Dunham, W. Schaff, and K. Smolenski, Appl. Phys. Lett. 103, 103504 (2013).

[12] S. Belomestnykh, M. Liepe, H. Padamsee, V. Shemelin, and V. Veshcherevich, High Average Power Fundamental Input Couplers for the Cornell University ERL: Requirements, Design Challenges and First Ideas (Cornell University, Ithaca, NY, 2008).

[13] H. Li, Multidimensional characterization of the laser and electron beams of the Cornell Energy Recovery Linac Photoinjector Prototype, Ph.D. thesis, Cornell University, 2012.

[14] Pulsar website for gpt, http://www.pulsar.nl/gpt/.

[15] Z. Zhao, B. M. Dunham, and F. W. Wise, J. Opt. Soc. Am. B 31, 33 (2014).

[16] Z. Zhao, A. Bartnik, F. W. Wise, I. V. Bazarov, and B. M. Dunham, Phys. Rev. ST Accel. Beams 17, 053501 (2014).

[17] D. Dowell (private communication). 